\title{
Mitteilungen der Van-Swienten-Gesellschaft
}

Hof rat-Erwin-Doman ig-Stipendium 1994

Die Van-Swieten-Gesellschaft - Verein Österreichischer Ärzte vergibt nach einem jährlich zu fassenden Vorstandsbeschhass - unter Berücksichtigung der jeweiligen finanziellen Gegebenheite $\prod$ - nach entsprechender Ausschreibung ein Stipendium, das nach dem Mitbegründer und Ehrenpräsidenten der Gesellschaft, Hofrat Erwin Do-manig, benannt ist.

Dieses Stipendium soil in erster Linie dem Kennenlerne $\prod$ und der praktischen Aneignung von neuen Methoden in der medizi [ischen Diagnostik und Therapie im Rahmen eines Auslandsauf $1 / 8$ nthaltes dienen; es darf nur an Nachwuchskräfte (Einzelpersonen) verliehen werden, die nach Werdegang und wissenschaftlicher Eignung i $\downarrow$ q Gewähr dafür bieten, dass ein im Interesse des medizinischen Fort-schrittes in Österreich fruchtbares Ergebnis zu erwarten ist. Die Ausschreibung erfolgt nach der 1. Vorstandssitzung des jeweiligen Präsidentenjahres durch das Sekretariat.

Die Bewerber richten, entsprechend den Ausschreibu Igsbedin-gungen, ihr Gesuch an das Sekretariat der Van-Swieten-Gesellschaft mit exakter Angabe des angestrebten Forschungsziels. Dem Gesuch sind beizulegen:

Curriculum vitae

Verzeichnis der wissenschaftlichen Publikationen

Detaillierte Angaben über die mit dem Stipendium verbundene Zielsetzung sowie über Ort, Zeit, Dauer und finanzielle Erforder-nisse

Empfehlung des Vorstandes der zuständigen Klinik ( $\Lambda$ bteilung, Institut)

Einreichungsberechtigt sind Nachwuchskräfte an österreichi-schen Kliniken, Abteilungen oder Instituten, welche im Besitz der österreichischen Staatsbürgerschaft und Mitglied der VanSwieten-Gesellschaft sein müssen.

Für die Vergabe des Stipendiums setzt der Vorstand der Van-Swieten-Gesellschaft ein vier- bis schsköpfiges Gremium unter dem Vorsitz des Präsidenten oder eines von ihm bestimmte Vertreters ein; dieses Gremium entscheidet mit Stimmenmehrheit unter Aus-schluss des Rechtsweges. Bei Stimmengleichheit steht dem Vorsit-zenden das Dirimierungsrecht zu. Nach Abschluss der geförderten Studien ist dem Vç > rstand der Van-Swieten-Gesellschaft ein schriftlicher Erfahrungsbericht zu er-statten; anlässlich der Jahrestagung der Van-SwietenQesellschaft hat der Stipendiat über Einladung des Präsidiums ein entSprechen-des Kurzreferat zu halten.

Anton-von-Eiselsberg-Preïs 1994

Der von der Van-Swieten-Gesellschaft gestiftete und gemeinsam mit der Österreichischen Ärztekammer zur Verleihung gelangende Anton-von-Eiselsberg-Preis beträgt für das Jahr $1994 \mathrm{~S}$ 100000 .-und wird hiermit ausgeschrieben. Er ist in erster Linie für junge Kol-leginnen und Kollegen gedacht, da der Preis der Förderung des wissenschaftlichen Nachwuchses dienen soil. Österreichische Ärzte sind berechtigt, Arbeiten einzureichen. Die Arbeiten sollen die Ergebnisse eigener wissenschaftlicher, experi-menteller oder klinischer Untersuchungen aus einem Fachgebiet der Medizin zum Gegenstand haben. 
Bei Arbeiten, die nur im Manuskript vorliegen, muss der Nach-weis erbracht werden, dass sie von einer Fachzeitschrift zur Publika-tion angenommen wurden. Bereits publizierte Arbeiten können nur berücksichtigt werden, wenn sie nach dem 30. April 1993 erschienen sind.

Habilitationsschriften sowie Arbeiten, die schon für eine andere Preisausschreibung eingereicht wurden, sind von der Einreichung ausgeschlossen. Der eingereichten Arbeit (Original mit 5

Kopien) ist eine Erklärung des Autors beizulegen, dass alle an dem Zustande-kommen der Arbeit beteiligten wissenschaftlichen Mitarbeiter (ein-schliesslich Biologen, Chemiker, Psychologen, Statistiker usw.) im Titel oder in Fussnoten oder sonst in irgendeiner Weise genannt sind. Weiter muss diese Erklärung die Bestätigung enthalten, dass auch alle Mitautoren den Ausschreibungsbedingungen entsprechen. Klinik- und Institutsvorstände bzw. Primarärzte oder Leiter von Abteilungen (Departments) werden ersucht, selbst keine Arbeiten einzureichen. Wissenschaftlichen Arbeiten, welche nicht in deutscher, sondern in englischer Sprache erschienen sind, muss eine deutsche Zusammenfassung beigefügt werden.

Die Einreichung hat bis spätestens 1, Juni 1994 (Datum des Post-stempels) an den Sekretär der Van-Swieten-Gesellschaft, Univ.-Prof. Dr. E. Reinold, p.A. Österreichische Ärztekammer (Frau Homolka), Weihburggasse 10-12, 1010 Wien, zu erfolgen.

Ende der Einreichungsfrist: 30. Juni 1994

Gesuche sind zu richten an Herrn Univ.-Prof. Dr. J? Reinold, Sekretär der Van-Swieten-

Gesellschaft, p.A. ÖsterreichiSche Ärztekammer (Frau Homolka), Weihburggasse 10-12, 1010 $\mathrm{W}^{1} / \mathrm{sn}$. 